\title{
Chlorine inhalation produces nasal congestion in allergic rhinitics without mast cell degranulation
}

\author{
D. Shusterman*, J. Balmes*, P.C. Avila*, M.A. Murphy\#, E. Matovinovic*
}

Chlorine inhalation produces nasal congestion in allergic rhinitics without mast cell degranulation. D. Shusterman, J. Balmes, P.C. Avila, M.A. Murphy, E. Matovinovic. (C) ERS Journals Ltd 2003.

ABSTRACT: Seasonal allergic rhinitic (SAR) subjects are more sensitive to nasal irritants than nonrhinitic (NR) subjects; however, the mechanism underlying this difference is unclear. This study sought to determine whether irritant-induced nasal congestion involves mast cell degranulation.

Eight SAR and eight NR subjects were exposed to both 1.0 parts per million chlorine and filtered air in separate visits; exposures were via nasal mask and lasted 15 min. Rhinomanometry was performed before, immediately after and 15 min after exposure. Following $\geqslant 2$ weeks, exposures and symptom reporting were repeated with nasal lavage, rather than rhinomanometry, pre- and postexposure. A separate substudy using rye grass antigen provided a positive control. Mast cell tryptase was measured in nasal lavage fluid from both substudies using an automated fluoroenzyme immunoassay.

Chlorine provocation significantly increased nasal airway resistance in SAR but not NR subjects. Conversely, tryptase levels in nasal lavage fluid were unaffected. Nasal allergen challenge significantly increased both nasal obstruction and nasal lavage tryptase in SAR subjects.

Irritant-induced nasal congestion is more pronounced among seasonal allergic rhinitic than nonrhinitic subjects. However, unlike nasal allergen challenge, the mechanism of response to chlorine does not appear to involve mast cell degranulation.

Eur Respir J 2003; 21: 652-657.
*Dept of Medicine and ${ }^{\#}$ Dept of Pediatrics, University of California, San Francisco, CA, USA.

Correspondence: D. Shusterman, Upper Airway Biology Laboratory, University of California San Francisco, 1301 South 46th Street, Building 112, Richmond, CA 94804, USA.

Fax: 15102315636

E-mail: dennis@itsa.ucsf.edu

Keywords: Nasal irritation

neurogenic inflammation

rhinitis

rhinomanometry

tryptase

Received: June 62002

Accepted after revision: November 292002

This study was funded was provided by the National Institute of Environmental Health Sciences (R01 ES10424).
Upper respiratory tract complaints, including mucous membrane irritation, nasal congestion and rhinorrhoea, are among the symptoms most likely to be reported in polluted indoor environments [1-3]. Clinically, the term "building-related illness" has been applied to specific diagnoses associated with specific aetiologic agents in indoor air [4]. However, more often, nonspecific mucous membrane irritation is reported and airborne chemical agents are implicated, including environmental tobacco smoke (ETS), volatile organic compounds and reactive cleaning products (such as chlorine and ammonia). Terms such as "nonspecific building-related illness" or "sick building syndrome" are often used to describe such situations, and symptom reporting may be disproportionate among individuals with pre-existing allergic disorders [3-9]. Consistent with these findings, the focus of the present research is the role of allergy as an apparent marker of nasal reactivity to air pollutants.

Despite similarities between irritant-associated upper respiratory tract symptoms and manifestations of allergic rhinitis, evidence points to nonallergic mechanisms in their pathogenesis. For example, BASCOM and co-workers [10, 11] showed that subjects who gave a history of reactivity to ETS tended to congest more with experimental ETS exposure than controls. However, the same subjects did not show evidence of mast cell degranulation (histamine, kinins, tosyl-L-arginine methyl esterase) or plasma leak (albumin) in nasal lavage fluid when provocation was repeated. The authors concluded that nonallergic (neurogenic) mechanisms may be operative in the congestive response to nasal irritant provocation [10, 11].

Previously, the authors utilised chlorine gas (0.5-1.0 parts per milliom (ppm) in air administered by nasal mask) as a model irritant for the upper airway and have documented nasal congestion using rhinomanometry. Using this experimental system, the authors showed that subjects with seasonal allergic rhinitis (SAR) nasally congest more after chlorine inhalation than nonrhinitic (NR) subjects, and that this differential response was not affected by cholinergic blockade $[12,13]$. In order to better understand the mechanism(s) involved in the nasal congestive response to chemical irritants, the authors intended: 1) to confirm that the lack of mast cell degranulation, previously demonstrated for ETS-associated nasal congestion, extends to a different class of chemical agents; and 2) to do so in the context of subjects stratified for differential sensitivity by an objective marker (i.e. presence or absence of nasal allergies). The authors consequently exposed 16 subjects, evenly divided by sex and allergy status, to dilute chlorine gas, documented irritant-related congestion, then repeated the experiment on the same subjects, analysing for tryptase in nasal lavage fluid as a marker of mast cell activation. Included in the study was an allergen provocation substudy ("positive control") to verify the sensitivity of the authors tryptase assay.

\section{Materials and methods}

\section{Study subjects}

Subjects were recruited through posters and newspaper advertisements. Inclusion criteria were aged 18-69 yrs and "general good health". Exclusion criteria were: 1) a history of asthma; 2) cigarette smoking (active or within previous 
6 months); 3) pregnancy or lactation; 4) a history of severe allergic reactions (anaphylaxis or angioedema); and 5) continuous therapy with medications having antihistaminic side effects (e.g. tricyclic antidepressants). Subjects read and signed an informed consent document approved by the Committee on Human Research of the University of California, San Francisco. Questionnaires were administered to each potential subject, who was provisionally classified as having SAR, NR, or "other" (including perennial allergic rhinitis).

Allergy skin-prick tests (to 13 regionally common aeroallergens/ mixes, plus saline and histamine controls) were then administered. For purposes of this study, SAR subjects were defined as subjects having: 1) a history of seasonally occurring sneezing, nasal pruritus, rhinorrhoea, postnasal drip, and/or nasal congestion, with or without known precipitants; and 2) skin-test reactivity to at least one seasonally occurring agent from the panel that corroborated the history. Skin-test reactivity was defined as a weal reaction to skin-prick testing with a diameter at least that of the histamine control. Nonrhinitics were defined as subjects who reported, at most, infrequent nasal symptoms without identified seasonal variation or precipitants, significant skin-test reactivity to no more than one agent in the panel of 13 aeroallergens, and normal findings on anterior rhinoscopy. Prior to skin testing, subjects were asked to refrain from taking antihistamines for $72 \mathrm{~h}$ (hydroxyzine for 3 weeks, astemizole for 12 weeks).

\section{Study design}

The study design was experimental, utilising a semirandomised crossover design comparing the effect of dilute chlorine gas with that of air (fig. 1). Two different end-points, nasal airway resistance (NAR) by active posterior rhinomanometry and mast cell tryptase concentrations in nasal lavage fluid, were ascertained in separate subexperiments in order to avoid artefactual effects of lavage on NAR. Each chlorine provocation subexperiment involved the same 16 subjects, eight SAR and eight NR controls, which were tested on a total of four occasions each. The concentration and duration of chlorine exposure, $1.0 \mathrm{ppm}$ for $15 \mathrm{~min}$, is the USA occupational shortterm exposure limit and, hence, is of both scientific and regulatory interest. SAR and NR subgroups were evenly divided by sex. In addition to undergoing chlorine provocation, six SAR subjects, plus an additional four recruited specifically for the substudy, underwent nasal allergen challenge, the purpose of which was to provide a positive control for the tryptase analytic method. At least 2 weeks separated

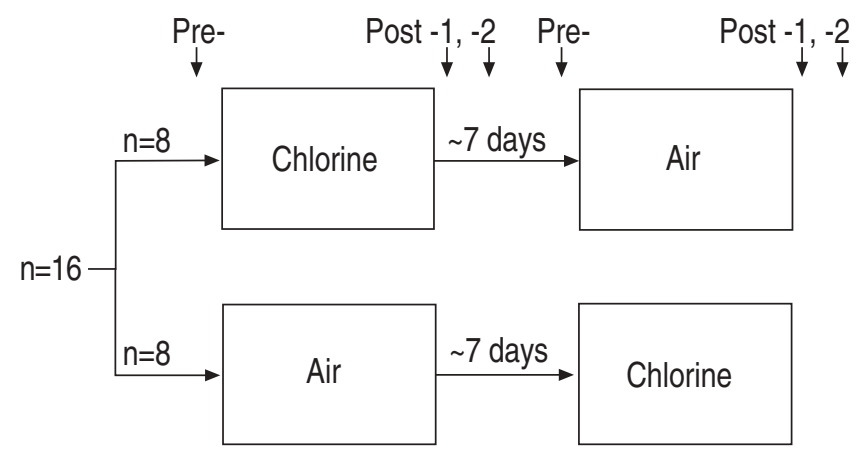

Fig. 1.-Structure of the chlorine provocation subexperiments involving 16 seasonal allergic rhinitic and nonrhinitic subjects. Sensory rating and nasal airway resistance measurements were taken pre- or post- (1: immediately after; 2: $15 \mathrm{~min}$ after) exposure. At least 2 weeks later sensory rating and nasal lavage measurements were taken at the same time points. The subexperiments were counterbalanced with respect to sex, rhinitis status and order of exposure. subjects' participation in the various subexperiments in order to avoid stimulus carry-over effects.

\section{Methods}

Chlorine provocation experiment. The first pair of exposures (1 week apart) involved rhinomanometry, with nasal airway resistance measured at baseline, immediately after and again 15-min postexposure. The second pair of exposures, conducted $\geqslant 2$ weeks later, involved nasal lavage, again performed at baseline, immediately after and 15-min postexposure. On a given day, exposure was either to pure (medical grade) air or to chlorine $(1.0 \mathrm{ppm})$ diluted in air. The order of exposure within each pair of testing dates was determined by limited randomisation (with an equal number of subjects being exposed to either air or chlorine first).

SAR subjects were tested out of their relevant pollen season. All subjects were asked to avoid exercising, consumption of spicy foods and use of scented cosmetics on the day of testing. In addition to antihistamine preclusions (as specified for skin-prick testing), subjects were asked to avoid using nasal steroids for $\geqslant 2$ weeks and nasal decongestants for $\geqslant 48 \mathrm{~h}$ prior to testing. Upon arrival at the laboratory, subjects entered a climate-controlled chamber $\left(22 \pm 1^{\circ} \mathrm{C}, 40 \pm 3 \%\right.$ relative humidity) with filtered air (activated charcoal and high-efficiency particulate). After a 15 -min waiting period, baseline symptoms (nasal irritation, nasal congestion, rhinorrhoea, postnasal drip and odour) were rated on computerbased visual analog scales (LabView software; National Instruments, Austin, TX, USA). The scales were indexed at equal intervals with the words "none", "slight", "moderate", "strong", "very strong" and "overpowering" corresponding to the numerical range of $0-5$. Symptom rating was repeated at the end of the 15-min exposure period and again 15-min postexposure.

For both subexperiments, chlorine or air was administered on a single-blinded basis for a period of $15 \mathrm{~min}$. Chlorine was diluted in medical-grade air, which was preconditioned to $22^{\circ} \mathrm{C}$ and $40 \%$ relative humidity; the dilution apparatus has been described in detail previously [12]. Chlorine concentrations were measured in real time using an electrochemical monitor (Model 1340; Interscan Corp., Chatsworth, CA, USA). The meter was recalibrated on a daily basis using the certified contents of the chlorine cylinder as the standard. The gas mixture was administered to the subject through a nasal continuous positive airway pressure mask (Series 3121; Respironics, Inc., Murraysville, PA, USA), which was sized according to the individual subject. The combination of a high-flow rate $\left(60 \mathrm{~L} \cdot \mathrm{min}^{-1}\right)$ and a low-pressure gas scavenging system allowed subjects to breathe with negligible superimposed pressure or resistance. As a safety precaution, before the first NAR measurement or nasal lavage, and again at the end of the protocol, subjects performed three forced expiratory volume manoeuvres in a sitting position, without a nose clip, using a MicroLoop portable spirometer (Micro Medical Ltd, Kent, UK). The highest of three values of forced expiratory volume in one second and forced vital capacity were recorded for monitoring purposes, but were not further analysed as no systematic treatment-related effect had been observed in prior studies [12, 13].

Rhinomanometry subexperiment. Nasal airway resistance for each testing condition was taken as the mean of three values, as ascertained by active posterior rhinomanometry using a commercial instrument (Model NR6-2; GM Instruments, Kilwinnig, UK). NAR was calculated using the pressure cut-off method $(75 \mathrm{~Pa})$ and was obtained at baseline, immediately postexposure and 15-min postexposure on both chlorine and 
air days. Calibration procedures were employed as detailed previously [12]. The hypothesis to be tested was that SAR subjects would show a significantly greater increase in NAR over baseline than would NR controls, comparing chlorineversus air-exposure days.

Nasal lavage subexperiment. To obtain nasal lavage specimens, each nostril was slowly instilled with $2.5 \mathrm{~mL}$ of $37^{\circ} \mathrm{C} 0.9 \%$, sterile, pyrogen-free, nonbacteriostatic saline $(5 \mathrm{~mL}$ total). After a 10-s retention time, fluid was expelled into a cup. Two baseline samples were obtained on each subject, one before and one after "cleansing" lavage (a total of three 10$\mathrm{mL}$ boluses being used for that purpose). Two additional samples were obtained, one immediately after and the other 15 min after exposure (fig. 2). Samples were weighed, pipetted to homogenise, then centrifuged at $960 \times g$ for $15 \mathrm{~min}$. Mast cell tryptase levels were determined on the supernatant using

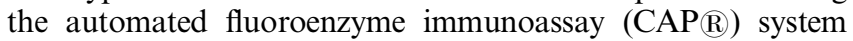
(Pharmacia-Upjohn, Kalamazoo, MI, USA). The hypothesis to be tested was that SAR subjects would show a significantly greater increase in tryptase over baseline (comparing chlorineversus air-exposure days) than would NR controls.

Allergen provocation (positive control). Allergen skin tests. To select the nasal allergen for nasal challenge, skin-prick testing was performed on 10 SAR subjects (six males) using five extracts: 1) Dermatophagoides pteronyssinus $\left(10,000 \mathrm{BAU} \cdot \mathrm{mL}^{-1}\right)$; 2) D. farinae $\left(10,000 \mathrm{BAU} \cdot \mathrm{mL}^{-1}\right)$; 3 ) cat hair $\left(10,000 \mathrm{BAU} \cdot \mathrm{mL}^{-1}\right)$; 4) rye grass $(2 \% \mathrm{w} / \mathrm{v})$; and 5$)$ birch tree $(2 \% \mathrm{w} / \mathrm{v})$. In addition, a negative control and histamine base $\left(1 \mathrm{mg} \cdot \mathrm{mL}^{-1}\right.$; Hollister-Stier Laboratories, Spokane, WA, USA) were used. The nasal allergen producing the largest weal was used for a skin prick titration, consisting of five-fold dilution steps of the selected nasal allergen in calcium/magnesium-free phosphate-buffered saline (PBS) from 1:5 to 1:78,125. All subjects had the largest reaction to the rye grass extract, which was consequently used for nasal challenge. The first dilution to elicit a negative skin response was the starting nasal allergen dilution in the nasal allergen challenge.

Nasal allergen challenge. First, the nasal cavities were rinsed with four nasal lavages. Then, the challenge started with diluent (calcium/magnesium-free PBS) followed every $15 \mathrm{~min}$ by increasing five-fold concentrations of rye grass allergen administered with an atomiser as described previously [14]. Ten minutes after each challenge the subject blew their nose into a collecting cup and graded (0: none; 1: slight; 2: moderate; 3) strong; 4) very strong; 5) overpowering) five nasal symptoms (congestion, irritation, itching, postnasal drip and runny nose). Nasal inspiratory peak flow (Youlten peak nasal inspiratory flow meter; Clement Clarke International Ltd, UK) and pulmonary expiratory peak flow (AirWatch;

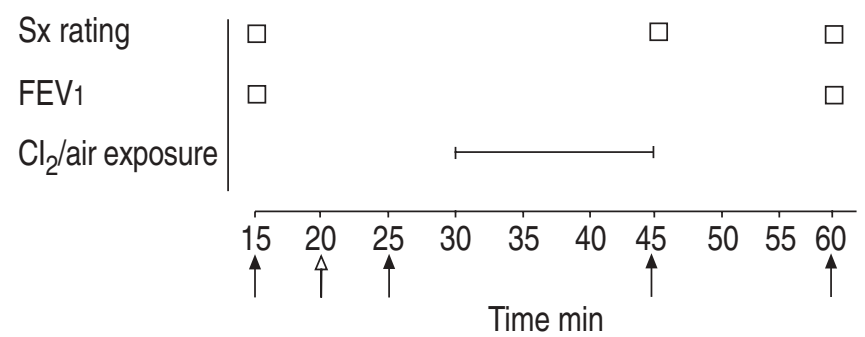

Fig. 2.-Chlorine $\left(\mathrm{Cl}_{2}\right)$ provocation/nasal lavage protocol. Sx: symptoms; FEV1: forced expiratory volume in one second. Closed arrows refer to application of a $5-\mathrm{mL}$ lavage and the open arrow refers to application of three $10-\mathrm{mL}$ "cleansing" lavages.
ENACT Health Management Systems, Palo Alto, CA, USA) were recorded as the best of three manoeuvres. Challenges progressed every $15 \mathrm{~min}$ until nasal inspiratory peak flow dropped by $\geqslant 50 \%$ from postdiluent value, or until pulmonary peak flow dropped by $\geqslant 20 \%$ [14].

The authors report on the analysis of three nasal lavage samples per subject, including prenasal allergen challenge (baseline), prenasal allergen challenge/post-PBS (control) and postnasal allergen challenge (performed $15 \mathrm{~min}$ after final allergen dose). Lavage samples were obtained and processed and mast cell tryptase levels were determined as described above for the chlorine provocation experiment.

\section{Analysis}

Based upon the authors' earlier work [12], a sample size of eight SAR and eight NR subjects providing $80 \%$ power was used to demonstrate a $20 \%$ difference in exposure-related nasal congestion between subgroups. In order to normalise different subjects' baseline NAR values (and to allow for dayto-day changes in baseline NAR for a given subject), proportional changes in NAR were studied throughout. This metric took the form of "proportional change in NAR" (from daily baseline) and "net proportional change in NAR" (chlorine minus air sessions), the latter being directly comparable between SAR and NR subjects. Symptom data were expressed as the observed change in numerical rating from the baseline rating given each testing day. For statistical purposes, tryptase results that were below the level of detection $\left(\right.$ i.e. $<1.0 \mu \mathrm{g} \cdot \mathrm{L}^{-1}$ ) were treated as one-half of the level of detection (i.e. $0.5 \mu \mathrm{g} \cdot \mathrm{L}^{-1}$ ).

For each statistical comparison, data were first examined for normality. Testing then proceeded using either analysis of variance or a nonparametric (i.e. Wilcoxon rank sum) test, as indicated. All tests were two-tailed, with statistical significance being defined as $\mathrm{p}<0.05$.

\section{Results}

\section{Subject recruitment}

A total of 20 subjects participated. Sixteen subjects, equally divided by sex and allergy status, completed the chlorine provocation substudies. Of these, six SAR subjects, along with another four SAR subjects recruited specifically for the purpose, participated in the allergen challenge. The characteristics of subjects are summarised in table 1 .

\section{Chlorine provocation study}

As a group, SAR subjects showed greater objective congestion (i.e. exposure-related increases in NAR) than did NR

Table 1.-Characteristics of participants

\begin{tabular}{|c|c|c|c|c|}
\hline & \multicolumn{2}{|c|}{ Chlorine provocation } & \multicolumn{2}{|c|}{ Allergen challenge } \\
\hline & Subjects $n$ & Age yrs & Subjects $n$ & Age yrs \\
\hline \multicolumn{5}{|l|}{ Males } \\
\hline Rhinitic & 4 & $30.3(21-39)$ & 6 & $28.9(21-40)$ \\
\hline Nonrhinitic & 4 & $33.5(23-51)$ & & \\
\hline \multicolumn{5}{|l|}{ Females } \\
\hline Rhinitic & 4 & $31.0(26-43)$ & 4 & $28.5(26-32)$ \\
\hline Nonrhinitic & 4 & $26.3(21-38)$ & & \\
\hline
\end{tabular}

Data are presented as mean (range) unless otherwise stated. 
controls, which actually tended to decongest after chlorine exposure (figs. 3 and 4). This difference reached statistical significance at 15 -min postexposure $(\mathrm{p}<0.05)$. However, symptomatic responses to chlorine were modest and did not differ significantly between SAR and NR subjects or from the response to air (data not shown). Chlorine inhalation did not produce an increase in tryptase levels, and in fact, all baseline and post-nasal lavage specimens were below the level of detection (i.e. $<1.0 \mu \mathrm{g} \cdot \mathrm{L}^{-1}$ ).

\section{Allergen provocation substudy (positive control)}

For all subjects, rye grass nasal allergen produced the largest skin-test reaction, and was utilised for nasal provocation purposes. A reduction in nasal inspiratory peak flow of $\geqslant 50 \%$ was achieved in all 10 subjects within the range of nasal allergen concentrations administered. Symptomatically, nasal allergen challenge produced significant increases over baseline for nasal irritation, congestion and rhinorrhoea (fig. 5). In terms of nasal lavage, all baseline (prenasal allergen challenge) tryptase levels were below the level of detection, whereas only 3 of 10 postnasal allergen challenge lavage specimens were undetectable. Nasal allergen challenge produced significant increases in mean tryptase levels over baseline in postchallenge specimens (fig. $6 ; \mathrm{p}<0.01$ by Wilcoxon rank sums). The statistical significance of this increase remained intact after exclusion of the two highest tryptase values, and after limitation of the analysis to the six subjects who underwent both nasal allergen challenge and chlorine provocation.

\section{Discussion}

Similarly to the authors' findings, two previous studies with SAR subjects showed an augmented tendency to congest nasally in response to irritant provocation (chlorine versus air) relative to control (NR) subjects. Repetition of the provocation protocol after a suitable interval failed to show evidence of chlorine-induced mast cell degranulation, as indicated by uniformly nondetectable tryptase levels in nasal lavage fluid pre- and postexposure. Moreover, the sensitivity of the assay

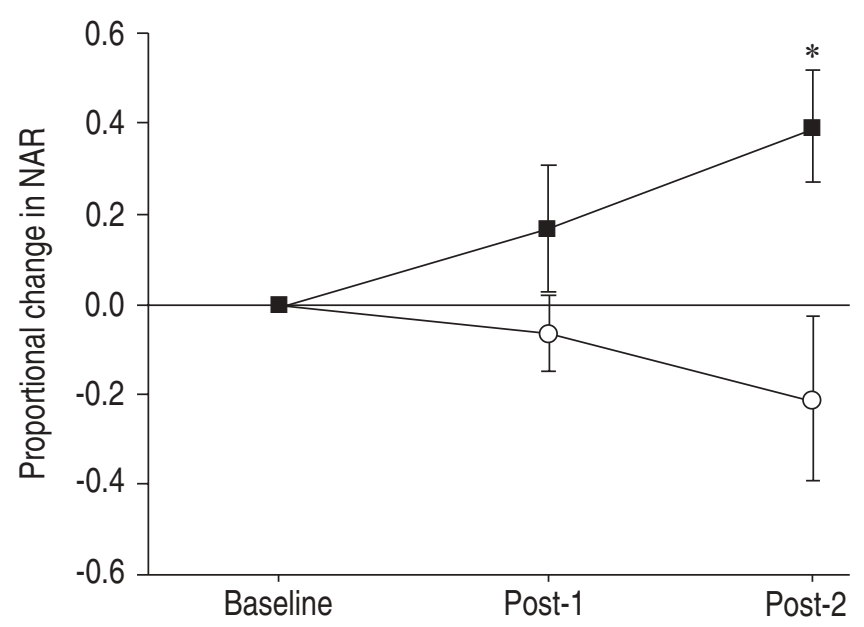

Fig. 3.-Chlorine provocation data showing net proportional change in nasal airway resistance (NAR) from baseline. Data are presented as mean \pm SEM. Post-1: immediately postexposure; Post-2: 15-min postexposure. [: seasonal allergic rhinitic subjects; $\bigcirc$ : nonrhinitic subjects. *: $\mathrm{p}<0.05$.
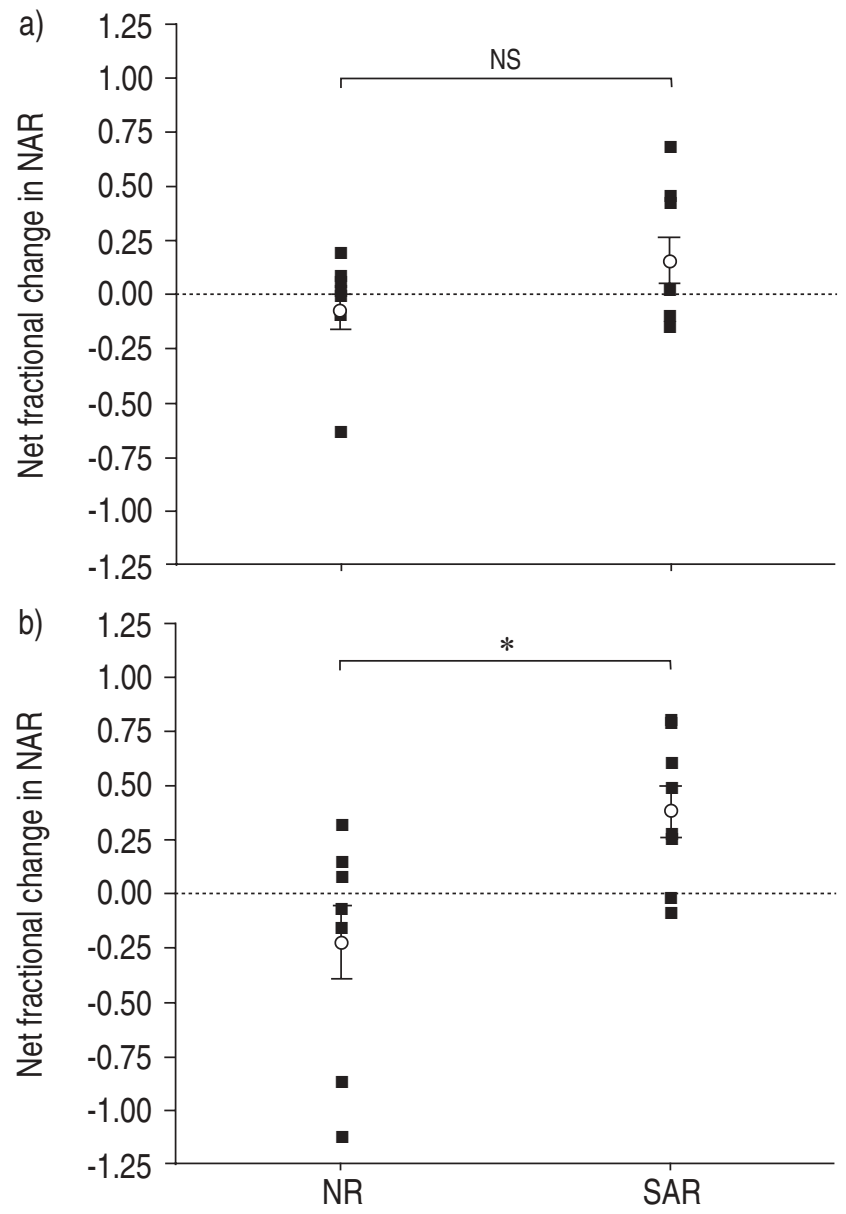

Fig. 4. - Chlorine provocation data showing individual net proportional change in nasal airway resistance (NAR) from baseline $(\bigcirc$ : mean \pm SEM). a) Post-1: immediately postexposure; b) Post-2: 15-min postexposure. NR: nonrhinitic subjects; SAR: seasonal allergic rhinitic subjects. NS: nonsignificant. *: $\mathrm{p}<0.05$.

was confirmed by nasally challenging a subgroup of SAR subjects with rye grass nasal allergen, producing both significant reductions in nasal inspiratory peak flow and significant postchallenge increases in tryptase. The results support the hypothesis that nonmast cell-mediated mechanisms, including possible neurogenic reflexes, may be operative in the nasal congestive response to irritants. Further, the results reconfirmed that the irritant-related nasal congestive response is augmented in the presence of allergic inflammation.

Consistent with previous findings of the authors, the symptomatic response to chlorine provocation was modest [12]. Ideally, a comparison of the biochemical response to chlorine and nasal allergen provocation would be matched for intensity of objective and/or subjective response. However, chlorine and nasal allergen provocation were carried out at separate physical sites, at which two different physiological measures, rhinomanometry and nasal inspiratory peak flow, were employed; tryptase analyses, by contrast, were all performed at the same facility. Furthermore, the two protocols differed fundamentally in that chlorine provocation employed a fixed dose, whereas nasal allergen challenge involved a variable dose titrated to a fixed end-point. Moreover, the potential for matching subjective end-points by using higher $\mathrm{Cl}_{2}$ exposure levels was effectively precluded by the fact that the concentration and time employed, $1.0 \mathrm{ppm}$ for $15 \mathrm{~min}$, is the occupational short-term exposure limit in the USA. Thus, the comparability of the two protocols is based upon the semiquantitative criterion that both involved 


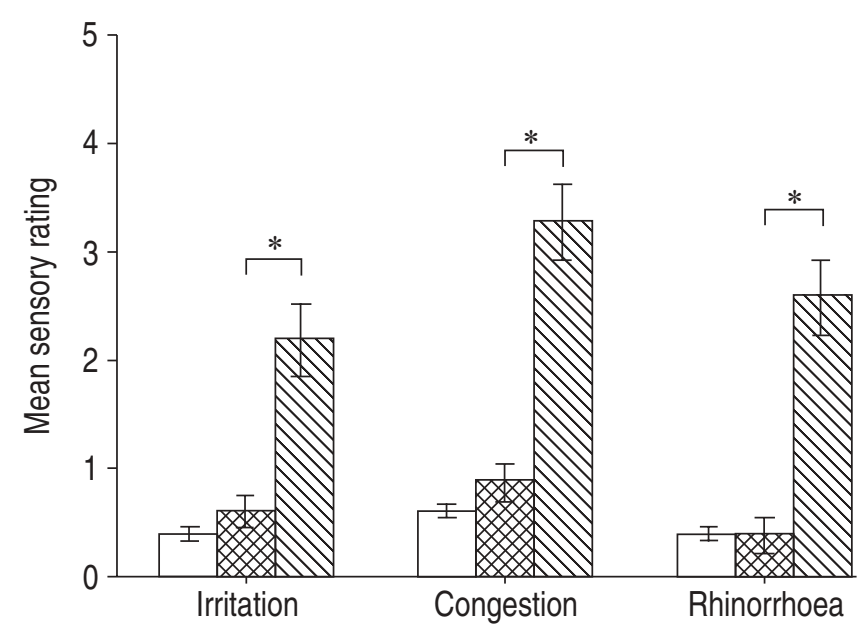

Fig. 5.-Allergen provocation data (mean \pm SEM) showing symptom rating for nasal irritation, congestion and rhinorrhoea at baseline $(\square)$, postphosphate-buffered saline challenge (control; a) and postnasal allergen challenge $(\mathbb{\mathbb { Q }})$. Rating scale 0 : none; 1: slight; 2: moderate; 3: strong; 4: very strong; 5: overpowering. *: $\mathrm{p}<0.05$.

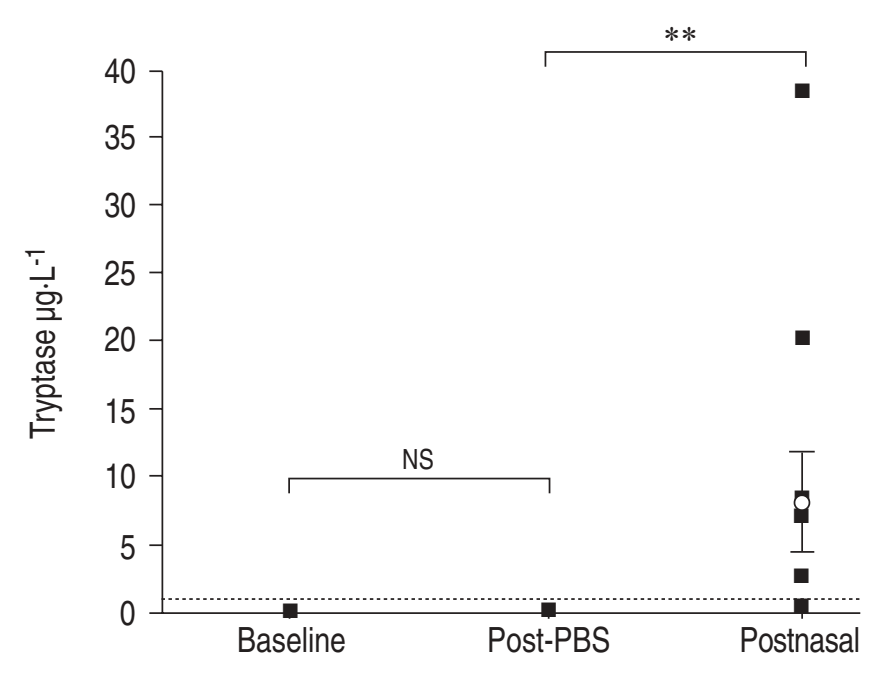

Fig. 6.-Allergen provocation data showing mast cell tryptase concentrations at baseline, postphosphate-buffered saline (PBS) challenge (control) and postnasal allergen challenge $(\bigcirc:$ mean \pm SEM $)$. ............ level of detection $\left(1.0 \mu \mathrm{g} \cdot \mathrm{L}^{-1}\right)$. NS: nonsignificant difference. $* *: \mathrm{p}<0.01$ compared with baseline.

significant objective nasal congestion (i.e. an increase in NAR or decrease in nasal inspiratory peak flow).

A novel finding in this, as compared with the authors' earlier studies, was that of a net decongestive effect of chlorine (versus air) exposure within the nonrhinitic (control) group. In two earlier studies by the authors, NR controls exhibited a neutral response to chlorine $[12,13]$. The finding in this case was driven, in large part, by the response of two individuals, one of whom showed progressive decongestion at postexposure times 1 and 2 (fig. 4). Future mechanistic studies may shed light on this interesting, but variably observed, phenomenon.

As noted above, one important finding was that of differential reactivity to irritant provocation by rhinitis status. The mechanism involved in augmented reactivity to chemical irritant stimuli in allergic rhintis is unknown, but several potential candidates exist. For example, in the likely event that neurogenic reflexes contribute to the nasal congestive response to irritants, then so-called "neuromodulation" by allergic mediators would be important. Neuromodulation has, in fact, been documented in other contexts. Using the model of a guinea-pig sensitised to a foreign protein (ovalbumin), RICCIO et al. [15] showed that antigen challenge of an ex vivo tracheal preparation reduced the threshold for mechanical stimulation required to produce a given frequency of afferent nerve impulses. Apparently, one or more product(s) of mast cell degranulation can acutely alter the sensitivity of afferent airway nerves to noxious stimuli. In addition, allergic modulation of efferent transmission through autonomic ganglia has been documented in the ovalbumin-sensitised guinea-pig model after acute allergen challenge [16].

In keeping with these physiological observations, some molecular targets of neuromodulation have been identified. Presynaptic muscarinic $\mathbf{M}_{2}$ receptors, for example, act as negative feedback elements within autonomic ganglia, and inactivation of $\mathrm{M}_{2}$ receptors results in increased synaptic transmission efficiency [17]. $\mathbf{M}_{2}$ receptor inactivation is not only an effect of major basic protein (derived from eosinophils), but is also produced by viral infection and ozone exposure, two other factors associated with airway hyperreactivity [18-20]. Similarly, nerve growth factor (NGF), which has been found preformed in both mast cells and eosinophils, has been documented at higher levels in nasal lavage fluid of allergic rhinitic versus control subjects [21]. NGF promotes synthesis of vasoactive neuropeptides (see below), which could also be involved in the nasal irritant response [22]. Both ex vivo and molecular biological studies, therefore, document potential neuromodulation by mediators of allergic inflammation.

Variations in responsiveness aside, the underlying mechanism of irritant-induced nasal congestion still remains in question. Given the findings reported here, downgrading the potential role of mast cell degranulation, along with findings by the author (and others) that cholinergic blockade does not diminish the nasal congestive response to irritants, the local (axon) reflex emerges as a credible mechanism for irritantinduced nasal congestion $[13,23,24]$. In animal experiments, neuropeptides have been implicated in the airway response to irritants in cigarette smoke [25]. Further, enhanced neuropeptide activity is found in allergic airways due to reduced levels of neutral endopeptidase [26]. Notwithstanding the biological plausibility of a neuropeptide-related mechanism in irritantinduced nasal congestion, however, direct evidence supporting this theory is lacking. This potential mechanism will constitute a focus of future studies by the authors.

Acknowledgements. Technical assistance was provided by J. Liu of the Asthma Research Center at the University of California, San Francisco.

\section{References}

1. Apter A, Bracker A, Hodgson M, Sidman J, Leung WY. Epidemiology of the sick building syndrome. J Allergy Clin Immunol 1994; 94: 277-288.

2. Fisk WJ, Mendell MJ, Daisey JM, et al. Phase 1 of the California Health Building Study: a summary. Indoor Air 1993; 3: 246-254.

3. Mendell MJ. Non-specific symptoms in office workers: a review and summary of the epidemiologic literature. Indoor Air 1993; 3: 227-236. 
4. Kreiss K. The epidemiology of building-related complaints and illness. Occup Med 1989; 4: 575-592.

5. Hodgson M. Sick building syndrome. Occup Med 2000; 15: 571-585.

6. Brasche $\mathrm{S}$, Bullinger $\mathrm{M}$, Morfeld $\mathrm{M}$, Gebhardt $\mathrm{HJ}$, Bischof W. Why do women suffer from sick building syndrome more often than men? Subjective higher sensitivity versus objective causes. Indoor Air 2001; 11: 217-222.

7. Cummings KM, Zaki A, Markello S. Variation in sensitivity to environmental tobacco smoke among adult non-smokers. Int J Epidemiol 1991; 20: 121-125.

8. Hall HI, Leaderer BP, Cain WS, Fidler AT. Personal risk factors associated with mucosal symptom prevalence in office workers. Indoor Air 1993; 3: 206-209.

9. Lundin L. Allergic and non-allergic students' perception of the same high school environment. Indoor Air 1999; 9: 92102.

10. Bascom R, Kulle T, Kagey-Sobotka A, Proud D. Upper respiratory tract environmental tobacco smoke sensitivity. Am Rev Respir Dis 1991; 143: 1304-1311.

11. Bascom R. Differential responsiveness to irritant mixtures. Possible mechanisms. Ann NY Acad Sci 1992; 641: 225-247.

12. Shusterman DJ, Murphy MA, Balmes JR. Subjects with seasonal allergic rhinitis and nonrhinitic subjects react differentially to nasal provocation with chlorine gas. J Allergy Clin Immunol 1998; 101: 732-740.

13. Shusterman D, Murphy M-A, Balmes J. Cholinergic blockade does not alter the nasal congestive response to irritant provocation. Rhinology 2002; 40: 141-146.

14. Avila PC, Abisheganaden JA, Wong $\mathrm{H}$, et al. Effects of allergic mucosal inflammation on the airway response to experimental rhinovirus 16 colds. J Allergy Clin Immunol 2000; 105: 923-932.

15. Riccio MM, Myers AC, Undem BJ. Immunomodulation of afferent neurons in guinea-pig isolated airway. $J$ Physiol 1996; 491: 499-509.

16. Myers AC, Undem BJ. Muscarinic receptor regulation of synaptic transmission in airway parasympathetic ganglia. Am J Physiol 1996; 270: 630-636.

17. Minette PA, Barnes PJ. Muscarinic receptor subtypes in lung. Clinical implications. Am Rev Respir Dis 1990; 141: $162-165$.

18. Evans CM, Fryer AD, Jacoby DB, Gleich GJ, Costello RW Pretreatment with antibody to eosinophil major basic protein prevents hyperresponsiveness by protecting neuronal $\mathrm{M}_{2}$ muscarinic receptors in antigen-challenged guinea pigs J Clin Invest 1997; 100: 2254-2262.

19. Fryer AD, Jacoby DB. Effect of inflammatory cell mediators on $\mathrm{M}_{2}$ muscarinic receptors in the lungs. Life Sci 1993; 52: 529-536.

20. Jacoby DB, Xiao HQ, Lee NH, Chan-Li Y, Fryer AD. Virus- and interferon-induced loss of inhibitory $\mathbf{M}_{2}$ muscarinic receptor function and gene expression in cultured airway parasympathetic neurons. J Clin Invest 1998; 102: 242-248.

21. Sanico AM, Stanisz AM, Gleeson TD, et al. Nerve growth factor expression and release in allergic inflammatory disease of the upper airways. Am J Respir Crit Care Med 2000; 161 1631-1635.

22. Togias A. Unique mechanistic features of allergic rhinitis J Allergy Clin Immunol 2000; 105: 599-604.

23. McLean JA, Mathews KP, Brayton PR, Solomon WR, Bayne NK. Effect of ammonia on nasal resistance in atopic and nonatopic subjects. Ann Otol Rhino Laryngol 1979; 88: 228-234.

24. Baraniuk JN. Sensory, parasympathetic, and sympathetic neural influences in the nasal mucosa. J Allergy Clin Immunol 1992; 90: 1045-1050.

25. Lundberg JM, Martling CR, Lundblad L. Cigarette smokeinduced irritation in the airways in relation to peptidecontaining, capsaicin-sensitive sensory neurons. Klin Wochenschr 1988; 66: Suppl. 11, 151-160.

26. Nadel JA. Regulation of neurogenic inflammation by neutral endopeptidase. Am Rev Respir Dis 1992; 145: 48-52. 